\title{
PROCRASTINACIÓN ACADÉMICA COMO PREDICTOR EN EL RENDIMIENTO ACADÉMICO EN JÓVENES DE EDUCACIÓN SUPERIOR
}

\author{
Academic procrastination as predictor of academic performance \\ among young people with higher education
}

Luis Alberto Chan Bazalar*

\begin{abstract}
Resumen
La finalidad del trabajo es presentar cómo la procrastinación o el posponer actividades académicas durante la formación del estudiante de educación superior, se relaciona con su rendimiento académico. De igual manera, se plantea un análisis cualitativo y cuantitativo en los aspectos: familiar, social, personal, logros y fracasos, yescolaridad. Además, se realiza un análisis en relación al rol genérico, familia, edad, lugar de nacimiento, entre otros.

Por lo tanto la importancia del estudio radica en la identificación de aspectos asociados a la procrastinación en jóvenes, y cómo ello puede incidir en su formación y desempeño pre-profesional.

La investigación se realizó con 200 estudiantes de educación superior de profesiones de letras en Lima; se aplicó una encuesta para la identificación de características de los participantes como también la Escala de Procrastinación Académica (Busko, 1998 y Álvarez, 2010-Adaptado en Lima). Se empleó un diseño descriptivo y correlacional para identificar relaciones y comparaciones, según rol genérico y rendimiento académico alto y bajo en sus estudios respectivos. Además, para una mayor apreciación especial se realizó un análisis de ítems. Los hallazgos obtenidos nos permiten identificar y discutir las diferentes situaciones que se asocian a la procrastinación en los estudiantes de educación superior y su posible repercusión en su rendimiento académico.

Los resultados nos permitirán presentar alternativas para la orientación y tutoría universitaria y que ello pueda incidir en la intervención psicoeducativa que se ejerce frente a los problemas de rendimiento académico de los jóvenes en el ámbito socio-educativo.
\end{abstract}

Palabras clave: Procrastinación, rendimiento académico, educación superior, estudiantes.

\begin{abstract}
The purpose of the paper is to present how procrastination or postponing academic activities during the formation of the higher education student is related to the academic performance. Similarly, a qualitative and quantitative analysis is brought up in the family, social, personal, achievements andfailures, and schooling aspects. Furthermore, is made an analysis in relation to the generic role, family, age, place of birth, among others.

Therefore, the importance of the study focus on the identification of aspects associated with procrastination in young people, and how this can affect their training and pre-professional performance.

The research was conducted with 200 higher education students of majors of letters in Lima; a survey for the identification of characteristics of participants as well as the academic procrastination scale was applied (Busko, 1998 and Alvarez, 2010-adapted in Lima).

A descriptive and co relational design was used to identify relationships and comparisons according to generic role and academic high and low performance in their respective studies. In addition, for a further special appreciation, an analysis of items was carried out. The findings obtained allow us to identify and discuss the different situations associated with procrastination in students of higher education and its potential impact on their academic performance.

The results will allow us to present some alternatives focus on the orientation and university tutoring, and this might affect the psycho educational intervention carried out in relation to the problems of academic performance of young people in the socio-educational field.
\end{abstract}

Keywords: Procrastination, academic performance, higher education, students.

* Psicólogo. Magíster y Doctor en Psicología. Docente del Departamento de Psicología. Universidad Femenina del Sagrado Corazón. lchanb@unife.edu.pe 


\section{INTRODUCCIÓN}

Ser estudiante hoy en día conlleva una serie de retos que debe enfrentar el joven que inicia o finaliza los estudios superiores en nuestro medio, y que claro está se presentan igualmente, a lo largo de toda su formación profesional asociados a circunstancias de índole familiar, social, económica, entre otras, sin dejar de lado los retos personales que engloban un conjunto de aspectos emocionales que en la mayoría de los casos no son resueltos y que por ende inciden sobre todo en el rendimiento académico, base de la formación del futuro profesional.

Uno de los desafíos que afrontan los estudiantes se relaciona a la procrastinación académica, término cuyo origen procede del latín procrastinare que textualmente significa "dejar para mañana". Históricamente, existen diversos tratados que aluden indirectamente a la procrastinación, como lo señala Steel (2007), quien se refiere al novelista inglés John Lyly, famoso por su obra Eupheus: The Anatomy of Wit, escrito en 1579, en el cual afirma: "Nada tan peligroso como la procrastinación" (p. 66). Asimismo, Steel refiere que un conde llamado Phillip Stanhope (1749/1968) aconsejó: "No a la ociosidad, no a la pereza, no a la postergación; no deje para mañana lo que puede hacer hoy" (p.66).

El presente estudio se fundamenta en los trabajos realizados originalmente por Busko (1998), quien considera que la procrastinación es una tendencia irracional a retrasar o evitar las tareas que deben completarse (estudio basado igualmente en los trabajos de Flett, Blankstein, Hewitt y Koledin en 1992, citados por Busko). Por otro lado diversos estudios se refieren a la naturaleza de las diferencias individuales en la procrastinación desde diversas perspectivas teóricas, debido a la sospecha de que este comportamiento se presenta externamente por una conexión entre la desidia y el perfeccionismo, relación que se puede examinar desde tres ángulos diferentes: primero, que la perfección influye en la procrastinación; segundo, que la procrastinación influye en el perfeccionismo; y tercero, que el perfeccionismo y la procrastinación se relacionan recíprocamente. Busko, además sostiene que la procrastinación tiene como partida la universidad o los estudios superiores.

Si bien es cierto que podemos estudiar la procrastinación desde diversas escuelas psicológicas, es pertinente tener en cuenta que ella posee una serie de características que tiene como base la responsabilidad, y hasta qué punto esto se inicia en aquellas pautas de crianza o educación por las que los jóvenes se sienten más atraídos por actividades más placenteras como el juego, la diversión u otras que los puedan llevar a algún tipo de adicción sea tecnológica o personal.

Por otro lado en los jóvenes la procrastinación afecta la toma de decisiones, ya sea para decidir si continuar con los estudios realizados o satisfacer los requerimientos que su entorno le exija, aun cuando su prestigio o dignidad se vea afectada por el murmullo o rumores acerca de su forma de ser.

Con base en los estudios realizados se han planteado tipologías de este perfil de personas, sin embargo todos ellos se refieren a dos estilos propiamente dichos: procrastinadores ocasionales $\mathrm{y}$ procrastinadores cotidianos, estos últimos son los que presentan problemas personales y de relación social.

Si el niño o el joven se inicia en la procrastinación, ello no solamente influirá en su entorno, en lo que le es específico, sino también en el hecho de generalizar "el dejar para mañana" otras actividades ya no solo académicas sino familiares, sociales, laborales, entre otras. De igual manera ello los llevaría a pensar que lo que hacen es lo correcto, y su frecuencia los puede incitar a buscar nuevos placeres reñidos por la sociedad o contrarios al bienestar psicológico, en una etapa la de ser estudiante- que abarca una serie de cambios tanto en los aspectos biológicos como psicológicos, funciones naturales propias del desarrollo humano. El hecho de ser "mayores de edad", de vivir la libertad de acción, es decir, los muchos cambios y experiencias nuevas que los jóvenes experimentan, les son más atrayentes que aquellas otras (experiencias) que por el momento no les brindan satisfacciones sino más bien tensiones o preocupaciones, las cuales pueden ser manejadas a través de la disculpa, engaños u otro tipo de explicaciones para justificar el hecho de no cumplir lo solicitado.

Es por esta razón que la procrastinación, en síntesis, viene a ser un problema de autocontrol y de organización del tiempo, lo que nos lleva a analizar cómo se instaura y qué alternativas debemos considerar para la superación de este comportamiento de "posponer actividades para más adelante", teniendo 
en cuenta un modelo que incluya una serie de aspectos que incidan sobre su forma de pensar.

Ental sentido nuestro trabajo se orienta a identificar y analizar cómo se presenta la procrastinación en estudiantes de letras de educación superior, según sus características psicosociales, y a vislumbrar la asociación con el alto o bajo rendimiento académico. Es pertinente considerar que el bagaje investigativo de las ciencias debe conllevar a una forma de intervención profesional resaltando aquellas directrices teóricas y aplicativas como las que propone Knaus (1997) en «Superar el hábito de posponer», estudio en el cual muestra una serie de características propias de las personas con tendencia a la procrastinación; entre ellas podemos mencionar: creencias irracionales, perfeccionismo, miedo al fracaso, ansiedad, rabia, impaciencia, necesidad de sentirse querido, sentirse saturado, resistencia al cambio, racionalización, impulsividad y mecanismos de huída. El trabajo concluye proponiendo alternativas como el resolver el hábito de posponer, empezar ahora y buscar frases positivas para sí mismo, escribir recordatorios y autoreforzarse constantemente.

\section{MÉTODO}

Los participantes fueron 200 estudiantes de educación superior del área de letras en Lima; la muestra fue seleccionada intencionalmente. Se aplicó una encuesta de características de los jóvenes como también la Escala de Procrastinación Académica (Busko, 1998 y Alvarez, 2010 - Adaptada a Lima). Se empleó un diseño descriptivo y correlacional para identificar relaciones y comparaciones, según rol genérico, semestres académicos, entre otros. Además, para una mayor apreciación se realizó un análisis de ítems. El estudio nos permite identificar y analizar las diferentes situaciones que se asocian a la procrastinaciónenlosestudiantes deeducación superior y su repercusión en el rendimiento académico.

\section{Tabla 1}

Edad

\begin{tabular}{lcc}
\hline & Frecuencia & Porcentajes \\
\hline 19 años & 53 & 26.5 \\
20 años & 34 & 17.0 \\
21 años & 41 & 20.5 \\
22 años & 29 & 14.5 \\
23 años a más & 43 & 21.5 \\
\hline
\end{tabular}

Con respecto a la edad de los participantes la mayoría tiene 19, 21 y 23 años de edad, lo que nos indica una proporción adecuada para su interpretación.

Tabla 2

Rol genérico

\begin{tabular}{llc}
\hline & Frecuencia & Porcentajes \\
\hline Masculino & 35 & 17,5 \\
Femenino & 165 & 82,5 \\
\hline
\end{tabular}

En lo que concierne al rol genérico las alumnas son la mayoría que fueron consideradas al azar cuando se aplicaron los materiales. 
Tabla 3

Lugar de nacimiento

\begin{tabular}{lrc}
\hline & Frecuencia & Porcentajes \\
\hline Lima (Capital) & 172 & 86,0 \\
Lima (Provincia) & 3 & 1,5 \\
Costa & 9 & 4,5 \\
Sierra & 9 & 4,5 \\
Selva & 1 & 0,5 \\
Extranjero & 6 & 3,0 \\
\hline
\end{tabular}

Los participantes casi en su totalidad respondieron haber nacido en Lima.

Tabla 4

Instrucción escolar

\begin{tabular}{lcc}
\hline & Frecuencia & Porcentajes \\
\hline Colegio Nacional & 4 & 2,0 \\
Colegio Particular Laico & 85 & 42,5 \\
Colegio Militar & 4 & 2,0 \\
Colegio Particular Religioso & 96 & 48,5 \\
Colegio Parroquial & 10 & 5,0 \\
Extranjero & 1 & 0,5 \\
\hline
\end{tabular}

En lo que se refiere al tipo de educación recibida en la secundaria el 91\% de los jóvenes manifestó haber estudiado en colegios particulares religioso y laico.

Tabla 5

Composición familiar

\begin{tabular}{lrc}
\hline & Frecuencia & Porcentajes \\
\hline Solo padres & 20 & 10,0 \\
Padres y Hermanos & 136 & 68,0 \\
Sólo hermanos & 8 & 4,0 \\
Familiares & 14 & 7,0 \\
Solo & 5 & 2,5 \\
Otro & 17 & 8,5 \\
\hline
\end{tabular}

Es relevante para el estudio que el $68 \%$ de participantes pertenezcan a familias constituidas por padres y hermanos. 
Tabla 6

\section{Repitencia escolar}

\begin{tabular}{lcc}
\hline & Frecuencia & Porcentajes \\
\hline Un curso & 26 & 13,0 \\
Más de un curso & 15 & 7,5 \\
Un año escolar & 8 & 4,0 \\
No repitió & 151 & 75,5 \\
\hline
\end{tabular}

La educación escolar es la base primordial para seguir estudios superiores, encontrándose que 49 participantes repitieron un curso, más de un curso o un año escolarlo que en términos porcentuales equivale a un $24,5 \%$

Tabla 7

\begin{tabular}{lcc}
\hline \multicolumn{3}{c}{ Repitencia de asignaturas en la educación superior } \\
\hline & Frecuencia & Porcentajes \\
Un curso & 38 & 19,0 \\
Dos o tres cursos & 52 & 26,0 \\
Cuatro o cinco cursos & 22 & 11,0 \\
Más de seis cursos & 11 & 5,5 \\
No repitió & 77 & 38,5 \\
\hline
\end{tabular}

Es anecdótico que los participantes, habiendo aprobado sus asignaturas en la educación escolar en un 24,5\%, ahora, en la educación superior aumentó a un $61.5 \%$.

Tabla 8

Rendimiento académico aprobatorio en el ciclo pasado

\begin{tabular}{lcc}
\hline & Frecuencia & Porcentajes \\
\hline 11 a 14 & 3 & 1,5 \\
15 a 17 & 54 & 27,0 \\
18 a 20 & 139 & 69,5 \\
No contestaron & 4 & 2,0 \\
\hline
\end{tabular}

En relación a la diversidad de asignaturas y formas de evaluación: el 98\% obtuvo notas aprobatorias en sus estudios. 
Tabla 9

Rendimiento académico desaprobatorio en el ciclo pasado

\begin{tabular}{lcc}
\hline & Frecuencia & Porcentajes \\
\hline 00 a 04 & 09 & 4,5 \\
05 a 07 & 35 & 17,5 \\
08 a 10 & 59 & 29,5 \\
Más de 11 & 93 & 46,5 \\
No contestaron & 4 & 2,0 \\
\hline
\end{tabular}

Si bien la educación superior presenta una estructura académica diferente a la educación escolar, se halla en los participantes que el $51,5 \%$ ha tenido dificultades en diversos cursos a nivel superior.

\section{RESULTADOS}

El análisis psicométrico de la Escala de Procrastinación Académica (véase tabla 10), reporta que los ítems se encuentran en un contínuum entre 0,20 y 0,68 , los cuales son significativos y aceptados estadísticamente (Kline, 1995). Igualmente encontramos que el material empleado presenta una confiabilidad adecuada.

\section{Tabla 10}

\section{Análisis de la confiabilidad de la Escala de Procrastinación Académica}

\begin{tabular}{rccc}
\hline Items & M & D.E. & Ritc* \\
\hline 1 & 2.92 & 0.99 & 0.68 \\
2 & 2.76 & 0.90 & 0.60 \\
3 & 2.88 & 0.97 & 0.44 \\
4 & 2.37 & 1.09 & 0.22 \\
5 & 2.35 & 0.94 & 0.20 \\
6 & 1.56 & 0.67 & 0.42 \\
7 & 2.30 & 0.86 & 0.60 \\
8 & 2.82 & 0.99 & 0.58 \\
9 & 2.89 & 0.96 & 0.60 \\
10 & 2.22 & 0.83 & 0.27 \\
11 & 2.49 & 0.90 & 0.57 \\
12 & 2.06 & 0.81 & 0.39 \\
13 & 2.44 & 0.92 & 0.58 \\
14 & 2.18 & 1.04 & 0.41 \\
15 & 2.87 & 0.91 & 0.41 \\
16 & 2.42 & 1.15 & 0.47 \\
\hline
\end{tabular}

Alfa de Cronbach $=0.84$

$* \mathrm{p}<0.05 \quad \mathrm{n}=200$

Por otro lado, al explorar la procrastinación según el rol genérico (véase tabla 11), hallamos que los varones tienden a procrastinar mayormente que las alumnas, sin embargo la diferencia no es distante, por lo que podríamos considerar que las estudiantes potencialmente estarían en camino a la procrastinación. 
Tabla 11

Análisis total de Procrastinación Académica según Rol Genérico

\begin{tabular}{lrc}
\hline Rol Genérico & N & Media \\
\hline Masculino & 35 & 43.4571 \\
Femenino & 165 & 38.6667 \\
\hline
\end{tabular}

Con respecto al rendimiento académico y procrastinación (véase tabla 12), estadísticamente los que "dejan para después" las actividades de estudio obtienen entre 15 y 17 de aprovechamiento (notas consideradas superiores) en relación a los que obtienen entre 11 y 14, o en todo caso, entre 18 a 20. Ello nos podría indicar que existen evaluaciones diversas en las cuales los estudiantes no pueden procrastinar debido a los temas de discusión, participación o aplicaciones prácticas sobre tos trabajos teóricos. Mientras que los alumnos que presentan dificultades (véase tabla 13), obviamente son los desaprobados que en nuestro trabajo representan un $51 \%$ del total de los participantes. Por lo que se podría decir que los comportamientos de procrastinación se presentan tanto en alumnos con notas superiores (15 y 17) como en aquellos con bajo rendimiento académico (notas 00 a 10 ).

Tabla 12

Análisis total de Procrastinación Académica según Calificaciones Aprobadas

\begin{tabular}{lrl}
\hline Calificaciones & N & Media \\
\hline 11 a 14 & 3 & 36.6667 \\
15 a 17 & 54 & 41.6667 \\
18 a 20 & 139 & 38.4964 \\
\hline
\end{tabular}

* No contestaron 4

Tabla 13

Análisis total de Procrastinación Académica según Calificaciones Desaprobadas

\begin{tabular}{lrl}
\hline Calificaciones & N & Media \\
\hline 00 a 04 & 9 & 43.5556 \\
05 a 07 & 35 & 41.1714 \\
08 a 10 & 59 & 39.5254 \\
\hline
\end{tabular}

* No contestaron 4 alumnos y 93 de ellos obtuvieron notas superiores a 11

A través del análisis de ítems (véase tabla 14), se ha podido establecer un perfil de características-situación de procrastinación de los participantes que indica que muchos de ellos presentan dificultades en su rendimiento académico: dejando sus tareas y estudio para el último momento, posponiendo la revisión de sus lecturas para otro momento, y, por otro lado, si no entienden los trabajos o clases no asisten a asesorías, faltan a clases, no presentan sus trabajos en las fechas indicadas, postergan trabajos y lecturas que les desagradan, no presentan motivaciones ni hábitos de estudio y cada vez que realizan alguna tarea la entregan sin la revisión previa. 
Tabla 14

Promedios porcentuales de Escala de Procrastinación Académica

\begin{tabular}{|c|c|c|c|c|}
\hline No. & Enunciados & Respuestas & fr. & $\%$ \\
\hline 01 & $\begin{array}{l}\text { Cuando tengo que hacer una tarea } \\
\text { normalmente la dejo para el último } \\
\text { minuto. }\end{array}$ & $\begin{array}{l}\text { Siempre } \\
\text { Casi siempre } \\
\text { A veces } \\
\text { Pocas veces } \\
\text { Nunca }\end{array}$ & $\begin{array}{r}8 \\
50 \\
77 \\
48 \\
17\end{array}$ & $\begin{array}{r}4 \\
25 \\
38.5 \\
24 \\
8.5\end{array}$ \\
\hline 02 & $\begin{array}{l}\text { Generalmente me preparo por } \\
\text { Adelantado para los exámenes }\end{array}$ & $\begin{array}{l}\text { Siempre } \\
\text { Casi siempre } \\
\text { A veces } \\
\text { Pocas veces } \\
\text { Nunca }\end{array}$ & $\begin{array}{r}6 \\
30 \\
90 \\
58 \\
16\end{array}$ & $\begin{array}{r}3 \\
15 \\
45 \\
29 \\
8\end{array}$ \\
\hline 03 & $\begin{array}{l}\text { Cuando me asignan lecturas, las leo } \\
\text { la noche anterior }\end{array}$ & $\begin{array}{l}\text { Siempre } \\
\text { Casi siempre } \\
\text { A veces } \\
\text { Pocas veces } \\
\text { Nunca }\end{array}$ & $\begin{array}{r}9 \\
45 \\
70 \\
64 \\
12\end{array}$ & $\begin{array}{r}4.5 \\
22.5 \\
35 \\
32 \\
6\end{array}$ \\
\hline 04 & $\begin{array}{l}\text { Cuando me asignan lecturas, las } \\
\text { reviso el mismo día de la clase }\end{array}$ & $\begin{array}{l}\text { Siempre } \\
\text { Casi siempre } \\
\text { A veces } \\
\text { Pocas veces } \\
\text { Nunca }\end{array}$ & $\begin{array}{r}6 \\
27 \\
53 \\
63 \\
51\end{array}$ & $\begin{array}{r}3 \\
13.5 \\
26.5 \\
31.5 \\
25\end{array}$ \\
\hline 05 & $\begin{array}{l}\text { Cuando tengo problemas para } \\
\text { entender algo, inmediatamente } \\
\text { trato de buscar ayuda }\end{array}$ & $\begin{array}{l}\text { Siempre } \\
\text { Casi siempre } \\
\text { A veces } \\
\text { Pocas veces } \\
\text { Nunca }\end{array}$ & $\begin{array}{r}5 \\
18 \\
52 \\
91 \\
34\end{array}$ & $\begin{array}{r}2.5 \\
9 \\
26 \\
45.5 \\
17\end{array}$ \\
\hline 06 & Asisto regularmente a clases & $\begin{array}{l}\text { Siempre } \\
\text { Casi siempre } \\
\text { A veces } \\
\text { Pocas veces } \\
\text { Nunca }\end{array}$ & $\begin{array}{r}1 \\
1 \\
12 \\
80 \\
105\end{array}$ & $\begin{array}{r}0.5 \\
0.5 \\
6 \\
40.5 \\
52.5\end{array}$ \\
\hline 07 & $\begin{array}{l}\text { Trato de completar el trabajo } \\
\text { asignado lo más pronto posible }\end{array}$ & $\begin{array}{l}\text { Siempre } \\
\text { Casi siempre } \\
\text { A veces } \\
\text { Pocas veces } \\
\text { Nunca }\end{array}$ & $\begin{array}{r}2 \\
15 \\
56 \\
95 \\
32\end{array}$ & $\begin{array}{r}1 \\
7.5 \\
28 \\
47.5 \\
16\end{array}$ \\
\hline 08 & $\begin{array}{l}\text { Postergo los trabajos de los cursos } \\
\text { que no me gustan }\end{array}$ & $\begin{array}{l}\text { Siempre } \\
\text { Casi siempre } \\
\text { A veces } \\
\text { Pocas veces }\end{array}$ & $\begin{array}{r}8 \\
39 \\
82 \\
50\end{array}$ & $\begin{array}{r}4 \\
19.5 \\
41 \\
25\end{array}$ \\
\hline
\end{tabular}


09 Postergo la lectura de los cursos que no me gustan

10 Constantemente intento mejorar mis hábitos de estudio

11 Invierto el tiempo necesario en estudiar aun cuando el tema sea aburrido

12 Trato de motivarme para mantener mi ritmo de estudio

13 Trato de terminar mis trabajos importantes con tiempo de sobra

14 Me tomo el tiempo de revisar mis tareas antes de entregarlas

15 Raramente dejo para mañana lo que puedo hacer hoy

16 Disfruto la mezcla de desafío con emoción de esperar hasta el último minuto para completar una tarea
Nunca

21

10.5

Siempre

9

4.5

Casi siempre

19.5

A veces

44.5

Pocas veces

Nunca

Siempre

Casi siempre

A veces

Pocas veces

Nunca

89

46

17

2

10

1

5

27

48.5

18.5

Siempre

1

Casi siempre

12.5

A veces

Pocas veces

Nunca

Siempre

Casi siempre

A veces

0.5

3

Pocas veces

24

Nunca

Siempre

1.5

Casi siempre

10.5

A veces

Pocas veces

33.5

Nunca

Siempre

2.5

Casi siempre

8.5

A veces

Pocas veces

24

Nunca

34.5

30.5

Siempre

Casi siempre

A veces

51

Pocas veces

20

Nunca

Siempre

Casi siempre

A veces

21.5

Pocas veces

35.5

Nunca 


\section{CONCLUSIONES}

1. Los estudiantes de educación superior participantes en este trabajo procrastinan comúnmente en diversas actividades académicas en su formación pre-profesional.

2. Los varones presentan mayor procrastinación que las alumnas en sus trabajos académicos.

3. El $61,5 \%$ de participantes que procrastina han desaprobado entre uno y seis cursos o materias durante el proceso de formación académica.

4. Por otro lado, un $68 \%$ de participantes que procrastinan pertenecen a familias compuestas por ambos padres e hijos.

5. La confiabilidad del material aplicado presenta un $84 \%$ de fiabilidad lo que determina una consistencia interna del trabajo realizado.

6. Los ítems-situaciones en los que mayormente procrastinan los participantes se refieren a: dejar las tareas para el último minuto, no estudiar antes para las evaluaciones, no cumplir con entregar sus trabajos, postergar trabajos o lecturas que no les agradan, incumplir con entregar los trabajos en fechas específicas, desmotivación y falta de hábitos de estudio.

\section{REFERENCIAS}

Alvarez, O. (2010). Procrastinación general y académica en estudiantes de secundaria de un grupo de colegios no estatales de Lima Metropolitana. Tesis para optar el Título de Licenciado en Psicología. Lima. Universidad de Lima.
Busko, D. (1998). Causes and consequences of perfectionism and procrastination: a estructural equation model (Tesis de maestría no publicada). Guelph, Ontario, University of Guelph.

Knaus, J. (1997). Superar el hábito de posponer. Revista de Toxicomanías, 13, 19-22. Recuperado de http://www.cat-barcelona.com/pdfret/RET133.pdf

Kline, P. (1995). The handbook of psychologycal testing. London: Routledge.

Riva, M. (2006). Manejo conductual cognitivo de un déficit en autocontrol, caracterizado por conductas de procrastinación. Tesis de Especialización en psicología clínica comportamental cognoscitiva. Bogotá. Pontificia Universidad Javeriana.

Rothblum, E. (1990). Fear of failure: the psychodynamic need achievement fear of success and procrastination models. Handbook of social and evaluation anxiety. New York: Leitenbreg.

Rothblum, E., Solomon, J. \& Murakami, J. (1986). Affective, cognitive, and Behavioral differences between high and low procrastinators. Journal of counseling psychology, 33, 387-394.

Stainton, M., Lay, C. \& Flett, G. (2000). Trait Procrastinators and Behavior/Trait-Specific Cognitions. Journal of Social Behavior and Personality, 15(5), 297-312.

Steel, P. (2007). The nature of procrastination: a metaanalytic and theoretical review of quintessential self-regulatory failure. Psychological Bulletin, 33(1), 65-85. 